\title{
Optical and Volumetric Relaxation Effects in Glass Following Removal of High Hydrostatic Pressures
}

\author{
C. Weir, S. Spinner, I. Malitson, and W. Rodney
}

\begin{abstract}
Optical and volumetric relaxations in glass were studied after compressing specimens 4 hours at 9,000 atmospheres and $21^{\circ} \mathrm{C}$. Following release of pressure the refractive index was higher than the original value and decreased with time, recovering its initial value within 12 hours. Optical-relaxation curves for lead and borosilicate glasses were complex, the detailed behavior depending on composition and thermal history. No corresponding effects were observed in fused silica after exposure to even higher pressures. Volumetric changes associated with index changes were determined interferometrically and found to be less than corresponding changes known to accompany annealing. Possible implications of the results are discussed.
\end{abstract}

\section{Introduction}

The delayed volumetric reaction of glass subjected to pressure was reported initially by Bridgman [1]. ${ }^{1}$ The first detailed study of the phenomenon was made by Bridgman and Simon [2], and was followed by experiments in which tensile stresses were combined with hydrostatic pressure [3]. The foregoing experiments indicated a threshold pressure below which compaction did not occur in most glasses [2]. Weir and Shartsis [4] measured the compressibility of binary alkali borate and silicate glasses up to 10,000 atm. They observed a nearly constant total decrease in volume of about 0.2 percent following compression. The maximum pressure was considerably below the reported thresholds for compaction of the alkali silicates [2], and the changes in volume were independent of composition. It was concluded that the effects observed were caused by loss of fragments of glass or penetration of the confining liquid into small crevices under pressure [4]. Anderson [5] studied the change in density at room temperature of glasses subjected to pressure at elevated temperatures for periods as long as 1 week. He found the process of density change at constant pressure to be complex and to consist of an initial reversible increase in density that approached a constant value after about $4 \mathrm{hr}$ and a subsequent irreversible increase in density that continued unabated for periods as long as 1 week. Bridgman, in a recent report [6], considers that no irreversible change in density should occur under purely hydrostatic compression and attributes his previous results [2] to the complex stress applied. He studied [6] a soda-silica glass under approximately hydrostatic conditions to $100,000 \mathrm{~kg} / \mathrm{cm}^{2}$ (about $97,000 \mathrm{~atm}$ ) and detected no change in dimensions on removal of pressure. Under the experimental conditions, however, very small changes could not have been detected.

Because evaluation of compressibility data on glasses requires some knowledge of the properties of a delayed volumetric response, it was considered desirable to ascertain the magnitude of this effect

1 Figures in brackets indicate the literature references at the end of this paper. in glasses subjected to pressures of the order of $10,000 \mathrm{~atm}$ at room temperature. It was decided to measure aftereffects of hydrostatic pressure, using the index of refraction as the indicating property, because it is known, on theoretical as well as experimental grounds, that the index is related to the density. The advantage of using the index lies in the fact that it can be measured with high precision, permitting the detection of small internal changes in the glass.

The data obtained by several workers $[7,8,9]$ show that the index changes linearly with density for the same glass on annealing. Analysis of the data of these workers [10], for a borosilicate glass (BSC 517) that was similar in composition to the ones used in the present study, shows the following empirical relationship between relative changes in index, $\Delta n / n$, and relative changes in density, $\Delta \rho / \rho$ :

$$
\Delta \rho / \rho=3.5 \Delta n / n \text {. }
$$

It can be shown from theoretical relationships between density and index $[11,12]$ that the numerical constant in eq (1) should have approximately the observed value, if the index is measured in a region of low dispersion. Such an analysis is given subsequently. Tool, Tilton, and Saunders [7] studied six glasses of widely different compositions, but the value of the numerical constant of eq (1) derived from their data was found to range only between 3.2 and 3.5 .

This report describes the optical relaxation observed in the index of refraction of glasses subjected to hydrostatic pressures of 9,000 atm at room temperature. Observations on other properties of interest, such as dimensions and absorption characteristics, were also conducted.

\section{Experimental Materials and Methods}

\subsection{Glasses}

Most of the studies were made by using a borosilicate glass designated as BSC 517/645. The weight percentages of the component oxides of the glass calculated from the batch composition were 
$\mathrm{SiO}_{2}, 66.4$ percent; $\mathrm{B}_{2} \mathrm{O}_{3}, 12.4$ percent; $\mathrm{Na}_{2} \mathrm{O}, 8.4$ percent; $\mathrm{K}_{2} \mathrm{O}, 11.8$ percent; $\mathrm{ZnO}, 0.5$ percent; and $\mathrm{As}_{2} \mathrm{O}_{3}, 0.5$ percent. Two specimens were cut from the same large sample. Different refractive indices (and densities) were obtained by applying different annealing procedures to each specimen. The bigher index resulted from annealing at $585^{\circ} \mathrm{C}$ for $12 \mathrm{hr}$ and cooling at a rate of $4 \mathrm{deg} \mathrm{C} / \mathrm{hr}$, whereas the lower index resulted from annealing at $590^{\circ} \mathrm{C}$ for $12 \mathrm{hr}$ and cooling at a rate of $50 \mathrm{deg} \mathrm{C} / \mathrm{hr}$. These annealing procedures are in general agreement with the procedures described by Lillie and Ritland. [13] for obtaining relatively strain-free specimens having a uniform index.

Additional experiments were performed on an experimental lead silicate glass consisting of 30 percent of $\mathrm{SiO}_{2}$ and 70 percent of $\mathrm{PbO}$ by weight as well as a specimen of fused silica of extremely high purity [14].

\subsection{Pressure Apparatus}

The apparatus and techniques used for obtaining high hydrostatic pressure have been described in detail [15]. Briefly, the glass specimens were immersed in a light petroleum distillate in the smooth bore of a heavy-walled pressure vessel. A leakproof piston, forced into the bore of the vessel by means of a hydraulic press, generated hydrostatic pressure by compressing the liquid. This pressure was measured with a manganin pressure gage mounted inside the vessel. A pressure of $9,000 \mathrm{~atm}$ was used in all experiments except one, which used 11,000 atm.

Pressure was applied at the rate of $1,000 \mathrm{~atm} / \mathrm{min}$ until the maximum pressure was attained. This pressure was maintained for $4 \mathrm{hr} \pm 1 \mathrm{~min}$ and released very rapidly. The pressure was dropped rapidly to $1,000 \mathrm{~atm}$, with a subsequent drop to 1 atm on disassembly of the pressure vessel. The entire operation of release of pressure and removal of the specimen required approximately $2 \mathrm{~min}$.

The specimens were chilled by the rapid decompression of the confining liquid and were transferred immediately to vacuum bottles containing water or petroleum distillate for transport to the laboratories in which the index and length measurements were made. The bottles and liquids were thermally equilibrated in the respective laboratories for at least $12 \mathrm{hr}$ prior to use. It was observed experimentally that a 4 - to 5-min immersion was sufficient to provide thermal equilibrium between the specimen and the excess liquid in the container. The pressure equipment itself was contained in a temperature controlled room at $21 \pm 1^{\circ} \mathrm{C}$.

\subsection{Refractive-Index Measurements}

The refractive indices of each prismatic sample of glass used in these experiments were measured on a precision spectrometer. The collimator and telescope have achromatic objective lenses of $250-\mathrm{mm}$ focal length and a $27-\mathrm{mm}$ clear aperture. The collimator is equipped with a bilateral variable slit, and the telescope has an Abbe-type autocollimating eyepiece. The telescope rotates on a cylindro-conical bearing.

The divided circle, approximately 3 in. in radius, is made of glass and graduated in intervals of $4 \mathrm{~min}$ of arc. The circle-reading microscope employs an optical coincidence micrometer with the eyepiece conveniently located below the telescope. Two diametrically opposite parts of the circle are simultaneously observed, and the optical micrometer permits direct readings to 0.1 sec of arc free of any error of eccentricity. The prism table, equipped with tangent screw and clamp, rotates on a vertical axis completely independent of the axis of rotation of the telescope. A removable table consisting of two slides that travel in superimposed ways at right angles to each other was constructed and mounted on the prism table to facilitate the proper centering: of the prism with respect to the collimator and telescope $[16,17]$.

Standard technique for high-precision refractiveindex measurements was carefully followed in these experiments. Measurements before and after compression showed no change in the refracting angles. Repetitive measurements were made at intervals over a period of $12 \mathrm{hr}$, with shorter intervals between measurements when the slope was greater. The indices were determined for two wavelengths, the 5461-A line of mercury and the 6438-A line of cadmium. All index measurements were carried out in a temperature-controlled laboratory, the temperature of which varied not more than $\pm 0.5 \mathrm{deg} C$ during the course of the measurements.

With a prism of refracting angle near $60^{\circ}$ and index near 1.5 , it has been calculated that an error of \pm 1 sec of arc in measuring the double-deviation angle leads to an error of $\pm 1.7 \times 10^{-6}$ in index. Any error in measurement of this prism angle does not affect the results, as long as the angle remains constant, because the main concern is the change in index.

It is estimated that the errors in measured angles do not exceed $\pm 0.75 \mathrm{sec}$ of arc or $\pm 1.2 \times 10^{-6}$ in index. This estimate is based on comparative measurements between the instruments used and another high-precision spectrometer and on measurements of the angles of standard prisms and stainless-steel wedges whose angles are estimated to be accurate to \pm 0.3 sec of arc.

\subsection{Dimensional Measurements}

The dimensional studies were made by using Saunders' [18] refinement of the interferometer method described by Peters and Cragoe [19]. The optical flats were separated by two specimens of the same glass, only one of which had been subjected to pressure. Each specimen was ground manually to contain three bosses for contact on the bottom flat, whereas the test specimen had a single boss and the comparison specimen two bosses for support of the upper plate. Monochromatic helium 5876-A light was used for illumination. The specimens used were $3 \mathrm{~cm}$ in height, and the flats were silvered to produce a sharpened fringe system. The lower flat was very close to the bottom surface of the upper flat and 
produced a true Fabry-Perot fringe system. This arrangement permitted counting of fractional fringes with a precision of about \pm 0.1 fringe.

It will be noted that a relaxation of the compressed specimen involves a tilting of the upper flat, and therefore requires a knowledge only of the total number of fringes. A variation in the ambient temperature produces only a translation of the entire fringe system.

\subsection{Transmission Measurements}

Absorption characteristics of the glasses were measured in transmission by using a manually operated commercial spectrophotometer. The specimens used were taken from samples of the glasses used for the index measurements.

\section{Results and Discussion}

\subsection{Optical Relaxation}

The measurements of index of refraction for different intervals following hydrostatic compression are shown graphically in figures 1 to 3 . Each figure shows data at wavelengths of 5461-A and 6438-A. The index of refraction prior to compression is represented by the point in each figure at zero time. Duplicate measurements were made on the two borosilicate glasses, and both sets of data are shown in figures 1 and 2 . These duplicates represent data obtained at different times on the same specimen. The dimensions of each point in the figures represent the estimated precision in index and time measurements.

Examination of the graphical results permits the following general conclusions:

a. A small but measurable relaxation in index of refraction follows the release of hydrostatic pressure on these glasses under the conditions of the experiments.

b. No permanent change in the index of refraction is observed, the value after about $12 \mathrm{hr}$ agreeing with the initial value within the experimental error.

c. The reproducibility of the data was generally within the experimental error in one case (fig. 2), whereas in the other (fig. 1), it was just outside the experimental error. Even in the latter case, however, curves obtained from either run retain their essential characteristics, so that any subsequent conclusions are unaffected by the differences.

d. The relaxation process is complex, consisting of two or more consecutive processes.

All curves show a rather rapid decay of index occurring in the first $1 / 2 \mathrm{hr}$, which is followed by at least one additional relaxation occurring after a considerably longer interval. The second relaxation, occurring at approximately $400 \mathrm{~min}$, is larger than the experimental error of the index determination only by a factor of about two. In addition, there is some uncertainty in the time base. It is conceivable then that the second relaxation may not be real if some unrecognized systematic error exists.

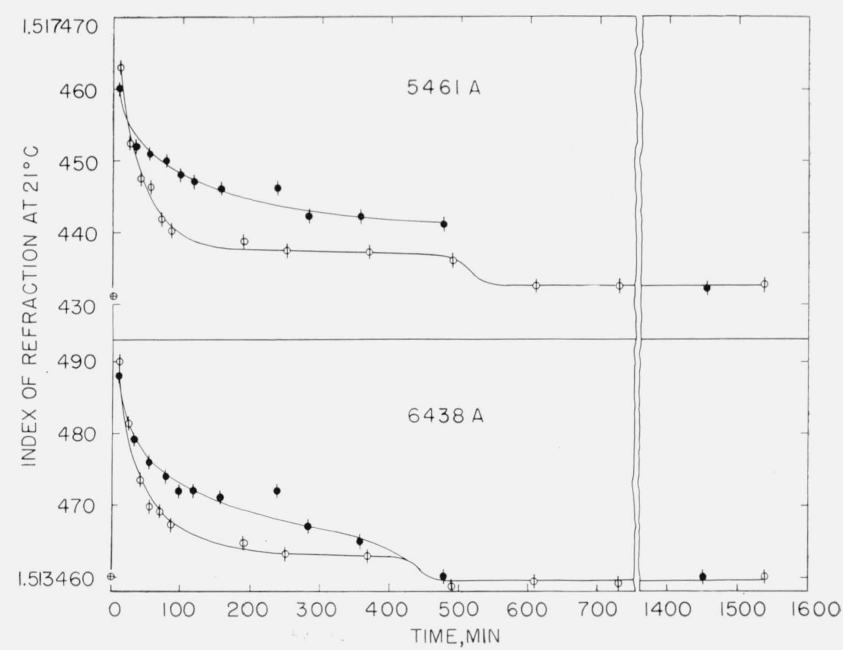

FIGURE 1. Isothermal relaxation in index at 1 atmosphere of specimen of borosilicate glass subjected to hydrostatic pressure of 9,000 atmospheres for 4 hours.

Initial density of glass, $2.513 \mathrm{~g} / \mathrm{cm}^{3}$

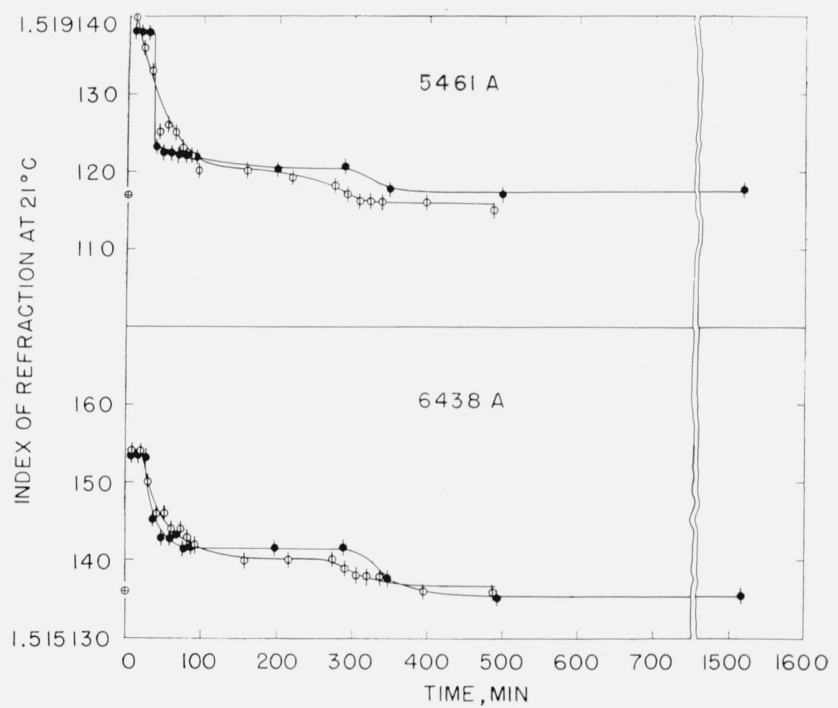

FIGURE 2. Isothermal relaxation in index at 1 atmosphere of specimen of borosilicate glass subjected to hydrostatic pressure of 9,000 atmospheres for 4 hours.

Initial density of glass, $2.530 \mathrm{~g} / \mathrm{cm} .^{3}$

Consideration of the precision of the measurements, as well as the fact that the phenomenon appears in each case, requires the conclusion that the second relaxation is probably real.

In the lead silicate glass, there appears to be a third relaxation process occurring, although the magnitude of the slowest of these is not appreciably beyond the experimental error. It is also interesting to note that only in this glass is there any substantial evidence that the relaxation processes occur at different times for the two wavelengths. This point is discussed later.

Because the two specimens of borosilicate glass studied were identical in composition, varying only 


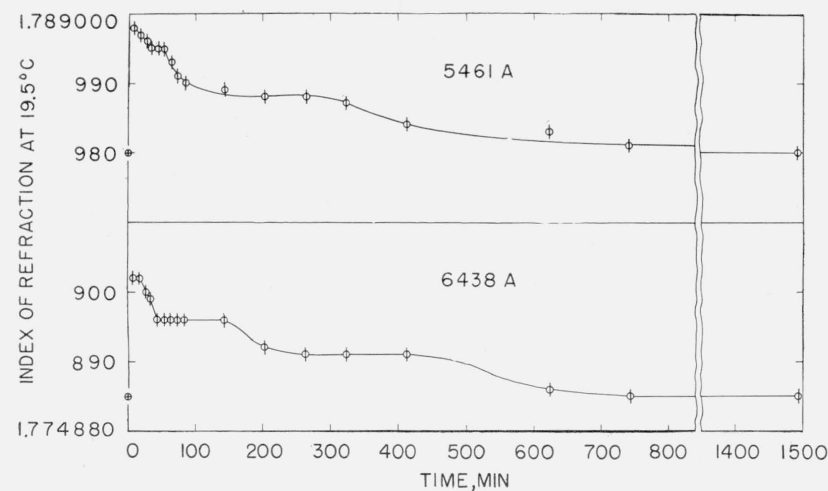

FIGURE 3. Isothermal relaxation in index at 1 atmosphere of specimen of lead silicate glass subjected to hydrostatic pressure of 9,000 atmospheres for 4 hours.

in their densities, it was expected that the less-dense specimen would show the greater effect after compression. This was actually observed, as shown in figures 1 and 2. The less-dense (lower-index) glass (fig. 1) shows a greater relaxation than the moredense (higher-index) glass shown in figure 2. It will also be observed that the over-all rate of relaxation appears to be related to the density. Both of these observations apply specifically to the initial relaxation, as the second effect is so small that no reliable conclusion may be drawn.

\subsection{Fused Silica}

Following the initial measurements on the borosilicate glasses, an attempt was made to observe relaxation in fused silica. Measurements made under the same conditions as those for the borosilicates showed no change in index. An additional experiment was performed by using a pressure of $11,000 \mathrm{~atm}$ for $6 \mathrm{hr}$. No change in index was observed under these conditions. It is concluded that within the experimental error of these measurements no optical relaxation occurs in fused silica in the time-pressure regions studied. This conclusion does not eliminate the possibility of relaxations occurring in less than the approximate $7 \mathrm{~min}$ required to obtain the initial measurement after release of pressure. With this reservation, the negative finding corroborates the report of Bridgman and Simon [2] of the marked threshold for the compaction of fused silica. The effect found for the borosilicates, coupled with the negative result for fused silica, pointed to the possibility that $\mathrm{B}_{2} \mathrm{O}_{3}$ was involved directly in the phenomenon because no threshold appears to exist for vitreous $\mathrm{B}_{2} \mathrm{O}_{3}$ [2]. To test this possibility the lead silicate glass that contained no $\mathrm{B}_{2} \mathrm{O}_{3}$ was tested; the relaxation observed eliminates the necessity of the direct involvement of $\mathrm{B}_{2} \mathrm{O}_{3}$.

\subsection{Effect of Ambient Temperature}

The precautions taken to maintain constant temperature during compression, index, and dimensional measurements, as well as during the transfer of the specimens, have already been mentioned. It is interesting to consider the effect of variations in temperature on the index and dimensional data.

The index of refraction of most glasses, including the types studied here, rises with increasing temperature in the vicinity of room temperature [20]. The value of the index-temperature coefficient is on the order of $2 \times 10^{-6} /{ }^{\circ} \mathrm{C}$. This means that in order to produce the observed initial index increase it would be necessary to assume a temperature rise of about $15 \mathrm{deg} \mathrm{C}$. This was impossible in view of the experimental precautions exercised.

The actual effect of rapid decompression is to chill the specimens. Therefore, any difference between the specimen temperature and the ambient temperature at the start of the index measurements would be in a direction to counteract the observed index effect. There is, indeed, a possibility that the horizontal portion of the curve observed at the onset in some of the figures could arise from these opposing tendencies.

In the case of the dimensional measurements, on the other hand, the effect of any initial cooling would be to augment the observed dimensional increase through the usual thermal expansion of the material occurring in the first few minutes of the observations. No such thermal effects were noted under the experimental conditions.

\subsection{General Considerations of Optical Relaxation}

All previous studies of glasses have resulted in reports of permanent increases in density $[2,3,5]$. Under the conditions of the present experiments, which used index as a measure of the condition of the glass, permanent changes were not observed. The permanent changes reported by Bridgman and Simon [2] may be attributed to the much higher pressures used or to the fact that the pressures used were not purely hydrostatic [6]. In Anderson's work [5], temperature was an added variable, but the pressures were somewhat lower than the 9,000 atm used here.

The index of refraction is related to both the density and the characteristic frequencies of the constituents of the glass $[11,12]$. The observed relaxation in index might arise from a relaxation in volume or in the change of characteristic frequencies or more probably a combination of both effects. The absorption properties of the glasses are of importance in considerations of optical relaxation.

\subsection{Optical Adsorption in the Glasses}

It has recently been reported that the ultraviolet absorption spectrum of benzene broadens and shifts to longer wavelengths on compression [21] and that a similar effect occurs in the infrared spectrum of deformed alkali halide crystals [22]. In the present experiments displacement of the electronic ultraviolet absorption region toward the measured wavelengths under pressure and a relaxation of the shift on removing the pressure would produce a result of type the observed. 
An experiment was conducted to detect a shift in the absorption edge of the lead silicate glass. No change in the position or shape of the absorption edge could be detected at $1 \mathrm{~atm}$ following compression at 9,000 atm for $4 \mathrm{hr}$. The precision of the absorption measurements was only of the order of a few parts in a thousand, and in view of the small change in index, the negative finding is not unexpected.

The absorption edge of the lead silicate glass is located at approximately $3500 \mathrm{~A}$. The glass was chosen for study for this reason, as well as the fact that it contained no $\mathrm{B}_{2} \mathrm{O}_{3}$, because wavelength dependence of the relaxation curves might be expected to be in evidence. The data of figure 3 indicate a difference in behavior at $6438 \mathrm{~A}$ and $5461 \mathrm{~A}$. From the previous considerations these differences may be indicative of absorption-band relaxation, although additional studies to corroborate the observation are required.

\subsection{Volume Relaxation}

Volume relaxation in gases and condensed phases is of considerable interest at the present time, with attention being concentrated on the subject principally by researches involving ultrasonics [23, 24, 25]. The relaxation in index observed here is doubtless accompanied by a related relaxation in volume, although in view of the foregoing discussion the two effects are not necessarily related linearly. As the glasses used here are considered to be isotropic and all changes are small, the relaxation in length may be considered to be one-third of the corresponding volume effect.

Studies of length changes were made only on the borosilicate glass of lesser density, as it would be expected to exhibit the largest effects. Using the ordinary interferometer system [19], any relaxation in length of the compressed specimen was so small as to be doubtful. From repeated experiments, however, it was concluded that a real relaxation occurred, which gave a value of $\Delta l / l$ of the order of $1 \times 10^{-5}$; On using a refined interferometer [18], a value of $1.2 \pm 0.3 \times 10^{-5}$ was obtained for $\Delta l / l$. Although the precision of the measurement was not adequate to determine reliable rate curves, it was observed that the general shape of the relaxation curve followed the index-time curve, with an initially rapid relaxation rate that decreased with time. From figure 1 the value of $\Delta n / n$ for this glass is observed to be $-2.0 \times 10^{-5}$; if eq (1) were valid for this process, a corresponding value for $\Delta l / l$ would be $2.3 \times 10^{-5}$. Thus the value of $1.2 \times 10^{-5}$ obtained experimentally for $\Delta l / l$ is approximately half that predicted by eq (1). It would appear that most of the probable systematic experimental errors (mainly arising from temperature) would tend to produce too low a value of $\Delta n / n$ and too large a value for $\Delta l / l$ because the thermal coefficients of index and length are positive [20] (sec. 3.3). It is concluded, therefore, that eq (1), which describes the index-density relationship on annealing, does not describe the processes occurring on volume relaxation in the glass studied.
The previous observations that the relative volume relaxation does not agree with that to be expected from the annealing relationship (eq 1) indicate that the relaxation effect involves shifts in the absorption frequencies in addition to the compaction (sec. 3.5). This concept may be developed in the following way: It may be assumed that the ordinary dispersion equations $[11,12]$ apply to these glasses. For the purposes of this discussion, similar results will be obtained from any of these equations and the Sellmeier equation is chosen for convenience. This relates the index, $n$, to the absorption wavelengths, $\lambda_{i}$, and the measured wavelength, $\lambda$, by the equation

$$
n^{2}=1+\sum_{i} \frac{A_{i} \lambda^{2}}{\lambda^{2}-\lambda i^{2}}
$$

where $A_{i}$ is proportional to the number of oscillators per unit volume. We are concerned with electronic absorption principally, and in the case of a glass the $\lambda_{i}$ will most probably consist of an average value of a distribution of individual $\lambda_{i}$ for each type of oscillator. Irrespective of the specific nature of the $\lambda_{i}$, on differentiation of eq (2), the following equation may be obtained:

$$
\frac{d n}{n}=\frac{1}{2 n^{2}}\left[\sum_{i}\left(\frac{A_{i} \lambda^{2}}{\lambda^{2}-\lambda_{i}^{2}} \frac{d A_{i}}{A_{i}}+\frac{2 A_{i} \lambda^{2} \lambda_{i} d \lambda_{i}}{\left(\lambda_{2}-\lambda_{i}^{2}\right)^{2}}\right)\right] .
$$

Provided $d A_{i} / A_{i}=-d V / V$, and in view of eq (2) ea (3) becomes

$$
\frac{d n}{n}=\frac{1}{2 n^{2}}\left[\left(1-n^{2}\right) d V / V+\sum_{i} \frac{2 A_{i} \lambda^{2}}{\left(\lambda^{2}-\lambda_{i}^{2}\right)^{2}} \lambda_{i} d \lambda_{i}\right] .
$$

The first term in brackets reflects the change arising from dimensional changes alone, whereas the second reflects that caused by shift in the $\lambda_{i}$. For glasses of index approximately 1.5 , the numerical coefficients of $d V / V$ reduce to 0.28 , which is approximately the reciprocal of the numerical coefficient of eq (1). If $\lambda_{i} d \lambda_{i} / \lambda^{2}-\lambda_{i}^{2} \ll 1$, the second term can be neglected, and eq (1) results from substituting $-d \rho / \rho$ for $d V / V$ and rearranging. If the present values of $d l / l$ and $d n / n$ obtained on relaxation are substituted in eq (4), it is not satisfied unless the absorptionshift term is retained. For these experiments it appears that the absorption-shift term and the volume-change term contribute about equally to the change in index. Conversely, it seems that on annealing, the change in index is almost fully accounted for by volumetric changes.

It is also of interest to note that increasing the density by lowering the temperature generally results in a decrease in index, indicating that the second term of eq (4) predominates [20].

\section{References}

[1] P. W. Bridgman, Proc. Am. Acad. Arts Sci. $\mathbf{8 1 ,} 165$ (1952)

[2] P. W. Bridgman add I. Simon, J. Appl. Phys 24, 405 (1953).

[3] P. W. Bridgman, J. Appl. Phys. 24, 560 (1953). 
[4] C. E. Weir and L. Shartsis, J. Am. Ceram. Soc. 38, 299 (1955).

[5] O. L. Anderson, J. Appl. Phys. 27, 943 (1956).

[6] P. W. Bridgman, Proc. Am. Acad. Arts Sci. 84, 111 (1955)

[7] A. Q. Tool, L. W. Tilton, and J. B. Saunders, J. Research NBS 38, 519 (1947) RP1793.

[8] N. J. Kreidl and R. A. Weidel, J. Am. Ceram. Soc. 35, 198 (1952).

[9] H. N. Ritland, J. Am. Ceram. Soc. 3886 (1955).

[10] S. Spinner and A. Napolitano, J. Am. Ceram. 39, 390 (1956).

[11] F. A. Jenkins and H. E. White, Fundamentals of optics, 2d ed. (McGraw-Hill Book Co., Inc., New York, N. Y., 1950).

[12] F. Seitz, The modern theory of solids (McGraw-Hill Book Co., Inc., New York, N. Y., 1940).

[13] H. R. Lillie and H. N. Ritland, J. Am. Ceram. Soc. 3y, 466 (1954)

[14] W. S. Rodney and R. J. Spindler, J. Research NBS 53, 185 (1954) RP2531; J. Opt. Soc. 44, 677 (1954).
[15] C. E. Weir, J. Research NBS 45, 468 (1950) RP2160.

[16] L. W. Tilton, BS J. Research 2, 909 (1929) RP64.

[17] R. J. Spindler and W. S. Rodney, J. Research NBS 49, 253 (1949) RP2361.

[18] J. B. Saunders, J. Research NBS 28, 51 (1942) RP1445.

[19] C. G. Peters and C. H. Cragoe, BS Sci. Pap. 16, 449 (1920).

[20] G. W. Morey, The properties of glass, 2d ed., p. 436 Reinhold Publishing Corp., New York, N. Y., 1954).

[21] W. Robertson, S. Babb, and F. Matsen, J. Chem. Phys. 26, 367 (1957).

[22] H. P. Lipson, E. Burnstein, and P. Smith, Phys. Rev. 99, 444 (1955).

[23] J. J. Markham, R. T. Beyer, and R. B. Lindsay, Rev. Mod. Phys. 23, 353 (1951).

24] E. G. Richardson, Rev. Mod. Phys. 27, 15 (1955).

[25] S. Kavim and L. Rosenhead, Rev. Mod. Phys. 24, 108 (1952).

Washington, November 2, 1956. 